\title{
Simulated Ion Trajectory and Induced Signal in Ion Cyclotron Resonance Ion Traps. Effect of Ion Initial Axial Position on Ion Coherence, Induced Signal, and Radial or $z$ Ejection in a Cubic Trap
}

\author{
Xinzhen Xiang* \\ Department of Chemistry, The Ohio State University, Columbus, Ohio, USA
}

\begin{abstract}
Alan G. Marshall
National High Magnetic Field Laboratory and Department of Chemistry, Florida State University. Tallahassee, Florida, USA
\end{abstract}

\begin{abstract}
The effects of ion initial axial position on coherence of ion motion, induced ion cyclotron resonance (ICR) signal, and radial and $z$ ejection have been evaluated by numerical simulation for a cubic Fourier transform-ion cyclotron resonance ion trap. For a given initial ion cyclotron phase and radius, ions of different initial $z$ position are shown to be excited to significantly different ion cyclotron radii (and ultimately radially ejected at significantly different excitation amplitude-duration products). Ion initial $z$ displacement from the trap midplane affects observed ICR signal magnitude in two ways: (1) for the same postexcitation cyclotron radius, an ion with larger initial $z$ displacement induces a smaller ICR signal and (2) an ion with larger initial $z$ displacement is excited to a smaller cyclotron radius. We also evaluate the induced ICR signal as a function of excitation amplitude-duration product for spatially uniform or Gaussian ion initial $z$ distributions. In general, if the excitation waveform contains components at frequency, $2 \omega_{z}$ or $\omega_{+}+2 \omega_{z}$, in which $\omega_{z}$ is the axial ("trapping") oscillation frequency, then ejection occurs axially. However, the resulting excitation amplitude-duration product for such axial ejection is significantly higher (factor of $\sim 4$ ) than that required for radial ejection (at $\omega_{+}$) for ions of small initial radius. The present results offer the first explanation of how, even if the ion is initially at rest on the $z$ axis (i.e., zero excitation electric field amplitude on the $z$ axis), $z$ ejection (axial ejection) may nevertheless occur if the excitation waveform contains frequency components at $\omega_{+}+2 \omega_{z}$ and/or $2 \omega_{z}$. Namely, our simulations reveal that off-resonant excitation pushes ions away from the $z$ axis, after which the ions are exposed to $z$ excitation and eventual $z$ ejection. (f Am Soc Mass Spectrom 1994, 5, 807-813)
\end{abstract}

$\mathrm{I}$ n Fourier transform-ion cyclotron resonance (FTICR) mass spectrometry, coherent ion cyclotron moLtion is excited by application of an if electric field oscillating at the (reduced) ion cyclotron frequency $\omega_{+}$ [1]. Between a pair of opposed detector electrodes, the coherently orbiting ion packet induces a detectable image charge whose magnitude oscillates at the ion cyclotron frequency [2-4]. An electronic current, which is the time derivative of the induced image charge, may be converted to a voltage signal that can be amplified and digitized. The detected signal in a typi-

Address reprint requests to Professor Alan G. Marshall, National High Magnetic Field Laboratory, Florida State University. 1800 East Paul Dirac Drive, Tallahassee, FL 32306-4005

Present address: The R. W. Johnson Pharmaceutical Research Institute, Welsh \& McKean Roads, Spring House, PA, 19477-0776. cal FT-ICR ion trap depends not only on the numbers and cyclotron orbital radii of ions of various mass-tocharge ratios, but also on the relative position of each ion within its orbiting packet, which is in turn determined by initial ion position and velocity and the frequency, amplitude, excitation mode (e.g., dipolar, quadrature, etc.), and duration of the excitation electric field. Nondestructive ion detection makes it possible to remeasure the same ions repeatedly for enhancement of signal-to-noise ratio [5-7], a major advantage over conventional electron multiplier detection. Detailed characterization of signal generation and detection is critical to improving quantitation of FT-ICR mass spectrometry.

The simplest excitation and detection electrode configuration consists of a pair of infinitely extended elec- 
trodes whose surfaces are parallel to the magnetic field $(z)$ direction. For this idealized model, the excitation electric field is spatially uniform and the detected signal magnitude is linearly proportional to the ion cyclotron radii and number of ions. Both excitation and detection processes are independent of ion location within the ion cyclotron resonance (ICR) ion trap. In this linear response limit $[8,9]$, the ICR signal magnitude is linearly proportional to the product of excitation period and excitation amplitude for single-frequency resonant excitation. Even when a quadrupolar electrostatic trapping potential is added, the equations of ion motion may still be solved analytically [10], and the initial spatial distribution within a packet of ions of the same mass-to-charge ratio does not change during on-off-resonant excitation [11].

However, in an actual (e.g., cubic) ICR ion trap, ICR response and detection are no longer linear processes, because the excitation and detection electric fields are no longer spatially uniform. Moreover, the trapping potential is not purely quadrupolar, and ICR frequency depends on ion position in the trap. As a result, (1) the ICR signal no longer varies precisely linearly with excitation electric field amplitudeduration product, (2) ions of different initial $z$ displacement from the trap midplane are resonantly (at $\omega_{+}$) excited to different cyclotron radii and are ultimately ejected radially but not simultaneously (see below), (3) ions may be ejected axially by dipolar radial excitation, if the excitation waveform contains frequency components at $2 \omega_{z}$ and $\omega_{+}+2 \omega_{z}$, in which $\omega_{z}$ is the axial ("trapping") oscillation frequency, and (4) Fourier transformation of the detected ICR signal yields

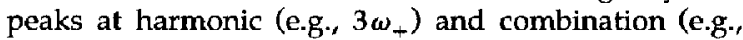
$\left.\omega_{+}-2 \omega_{z}\right)$ frequencies.

In prior treatments, the ion equations of motion have been solved for rf potential expanded up to fourth order in Cartesian coordinates. Such calculations show how ion loss due to $z$ ejection by dipolar radial excitation at $2 \omega_{z}$ and $\omega_{+}+2 \omega_{z}$ could arise from coupling of axial and radial motion during broadband excitation of initially off-axis ions [12-19]. However, in typical FT-ICR experiments, ions are formed on or very close to the central $z$ axis of the trap, where there is no radial excitation electric field component. Thus the origin of $z$ excitation under such conditions is not obvious. Moreover, there is presently little theoretical characterization of spatial coherence in an ion packet following spatially nonuniform excitation in a nonquadrupolar electrostatic trapping potential (e.g., a cubic ICR ion trap).

Dunbar [20] first applied the reciprocity principle (see below) to ICR Rempel et al. [13] derived an expression relating ion $z$ position and ICR signal magnitude. A comprehensive theory to derive the induced image charge on the detection electrodes by an orbiting ion [21] can be used to predict the frequencies and amplitudes of harmonic and side-band signals once an ion has been excited to a specified location and veloc- ity. None of the above treatments includes the excitation process in a real (e.g., cubic) trap. The purpose of this article is to present a systematic analysis of the effect of ion initial $z$ displacement from the trap midplane on ion cyclotron motion coherence, radial and axial ejection, and Fourier components of the induced ICR signal based on recently developed numerical simulation methods [22] for the commonly used cubic ICR ion trap.

\section{Computational Methods}

The calculation of ion trajectory and induced ICR signal for a single ion in a cubic trap has been described previously [22]. Here, the induced ICR signal for an ensemble of many ions of different initial axial displacement from the trap midplane is calculated as follows: For each individual ion at time $t$, the ion position $(x, y, z)$ and velocity $(\dot{x}, \dot{y}, \dot{z})$ are calculated from the initial ion position, velocity, and force acting on the ion. The current induced on detector electrodes is then obtained by reciprocity [21] and converted to a voltage based on an equivalent electronic circuit model $[2,4]$, in which the resistance $R$ is $10^{6} \Omega$ and the capacitance $C$ is $100 \times 10^{-12} \mathrm{~F}$. A composite transient time-domain signal is obtained from the superposition (sum) of the signals induced by each ion. Finally, a frequency-domain spectrum is generated from the Fourier transform of the composite time-domain signal. Ions initially are taken as evenly distributed along the $z$ axis or Gaussian distributed about the $z=0$ trap midplane:

$$
\frac{n(z)}{n}=\frac{1}{\sigma \sqrt{2 \pi}} \exp \left(\frac{-z^{2}}{2 \sigma^{2}}\right)
$$

in which $n(z)$ represents the number (out of a total of $n$ ) of ions displaced by distance $z$ from the trap midplane and the standard deviation, $\sigma=a / 6$, in which $a$ is the length of one side of the cubic trap, so that $99.7 \%$ of the ions are located within $-a / 2 \leq z \leq a / 2$. In other words, if the total number of ions is 100,000 , then 99,648 ions will be distributed within $\pm 3 \sigma$ of the trap midplane. The induced ICR signal has been computed for that case (see Results and Discussion). Other parameters for ion trajectory simulations throughout this article are: cubic trap of side length $a=1 \mathrm{in.}(2.54 \mathrm{~cm})$, that is, $-a / 2 \leq x \leq a / 2 ;-a / 2 \leq y \leq a / 2 ;-a / 2 \leq z$ $\leq a / 2$; ion mass-to-charge ratio $m / z=1000$ or 5000 in atomic mass units per elementary charge; ion initial kinetic energy 0; magnetic field induction $3.00 \mathrm{~T}$; electrostatic trapping voltage $1 \mathrm{~V}$ applied to each endcap electrode $(z= \pm a / 2)$; dipolar excitation voltage $10 \mathrm{~V}$ [peak to peak, $(\mathrm{p}-\mathrm{p})$ ] applied differentially between one opposed pair of side plates; computational time increment between data points $5 \times 10^{-9} \mathrm{~s} ; \omega_{+}$(for quadrupolar trapping) $=288,019.792 \mathrm{rad} / \mathrm{s} ; \omega_{-}$(for quadrupolar trapping) $=1440.263 \mathrm{rad} / \mathrm{s} ; \omega_{z}$ (for 
quadrupolar trapping) $=28,803.619 \mathrm{rad} / \mathrm{s}$; detection circuit equivalent capacitance $100 \mathrm{pF}$; detection circuit equivalent resistance $1 \mathrm{M} \Omega$.

\section{Results and Discussion}

\section{Effects of z Displacement on Ion Coherence}

For spatially uniform excitation and a pure quadrupolar trapping potential, the ion relative positions inside an ion packet remain unchanged during either on- or off-resonance excitation [11]. However, in a finite-size (i.e., actual) ICR trap, the excitation electric field $x$ component $E_{\mathrm{x}}$ is largest in the $z=0$ trap midplane and decreases as the ion approaches either of the two trapping electrodes. Thus, an ion with a large initial $z$ displacement from the $z=0$ trap midplane oscillates in the $z$ direction about the trap midplane and on average is exposed to a lower amplitude electric excitation field (and is thus excited to a smaller cyclotron radius) than an ion that remains in trap midplane. Figure 1 shows trajectories for ions with small initial $z$ displacement (Figure 1a, $z<0.20 a$, where $a$ is the trap
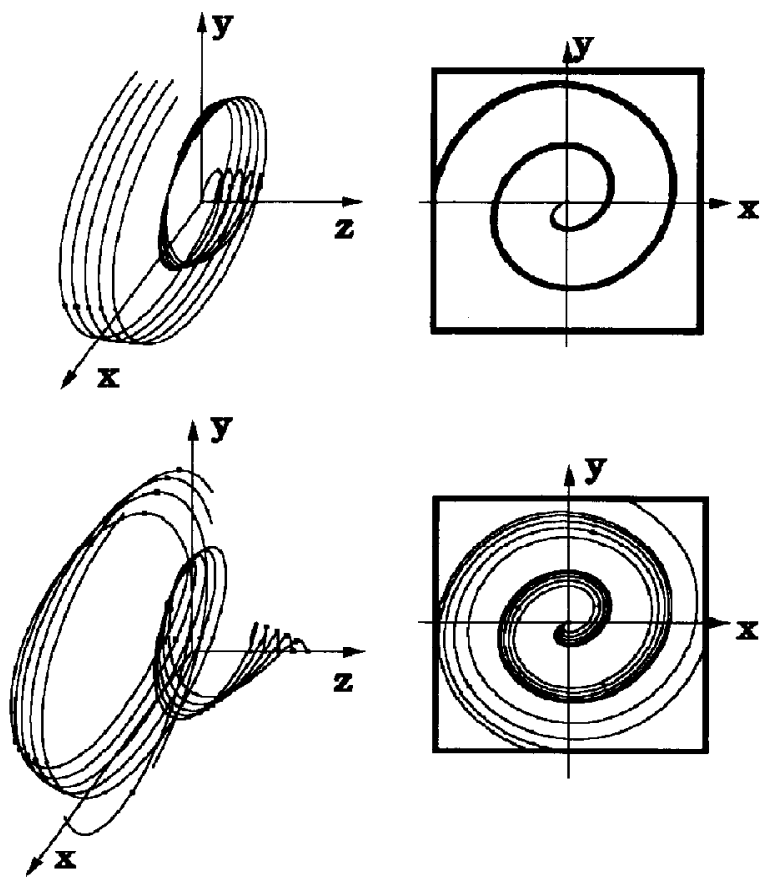

Figure 1. Trajectories during single-frequency on-resonance dipolar excitation for ions of different initial axial displacement $z(0)$ from the trap midplane. (a) Ions with small initial $z$ displacement $(0,0.05 a, 0.10 a, 0.15 a$, and $0.20 a)$, in which $a$ is the length of one side of a cubic ICR ion trap. (b) Large initial $z$ displacement $(0.20 a, 0.25 a, 0.30 a, 0.35 a$, and $0.40 a)$. In each case, ion $m / z 5000$, ion initial velocity is zero, and excitation electric field peak-to-peak amplitude is $10 / a \mathrm{~V} / \mathrm{m}$. Left: three-dimensional display; Right: projection of the ion trajectory onto the $z=0$ plane through the center of the trap. edge length) and large initial $z$ displacement (Figure $1 b, z>0.20 a$ ) during single-frequency on-resonance dipolar excitation. For initial $z$ displacement less than $\pm 0.20 a$, excited ions reach the $x$ (or $y$ ) electrodes approximately simultaneously. However, ions with initial $z$ displacement greater than $0.20 a$ have significantly smaller cyclotron radii, as seen from their trajectory projections onto the $z=0$ trap midplane.

Figure 2 is a plot of radial ejection onset (i.e., the length of time for a continuously resonantly excited ion to reach one of the side electrodes) as a function of ion initial $z$ displacement. Ejection time varies significantly with initial $z$ displacement: The ejection time for an ion initially at $z=0.45 a$ on the $z$ axis is approximately 1.5 times longer than that for an ion initially at rest at the trap center $(z=0)$. Interestingly, for dipolar radial excitation at the (reduced) ion cyclotron frequency $\omega_{+}$, ions are ultimately always ejected radially (rather than axially) no matter how large the excitation voltage.

Figure 3 shows a plot of instantaneous ion radius $r=\sqrt{x^{2}+y^{2}}$ (Figure 3a) and kinetic energy (Figure 3b) during dipolar resonant excitation. In the linearresponse model for spatially uniform excitation, ion radius increases linearly with excitation period for ions at any position. In the cubic trap, the excitation field (and ion cyclotron frequency and $z$ oscillation frequency) is position dependent and thus the excited ion radius as a function during excitation is modulated at both the axial and magnetron frequencies. (The magnetron modulation is not evident in Figure 3 because

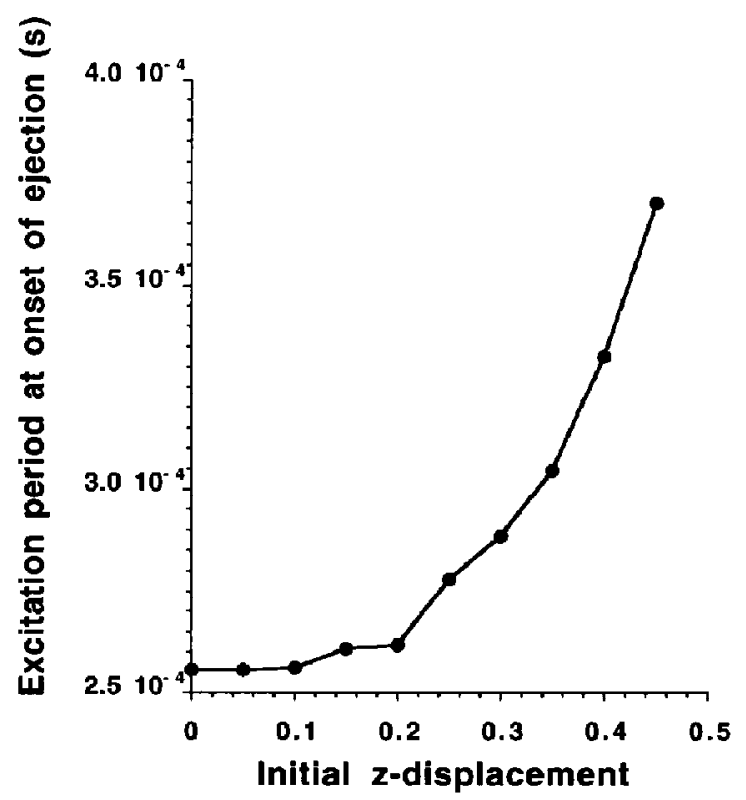

Figure 2. On-resonance single-frequency excitation period at onset of radial ejection as a function of initial $z$ displacement for ions of $m / z 1000$ initially located on the $z$ axis of the ICR ion trap. 

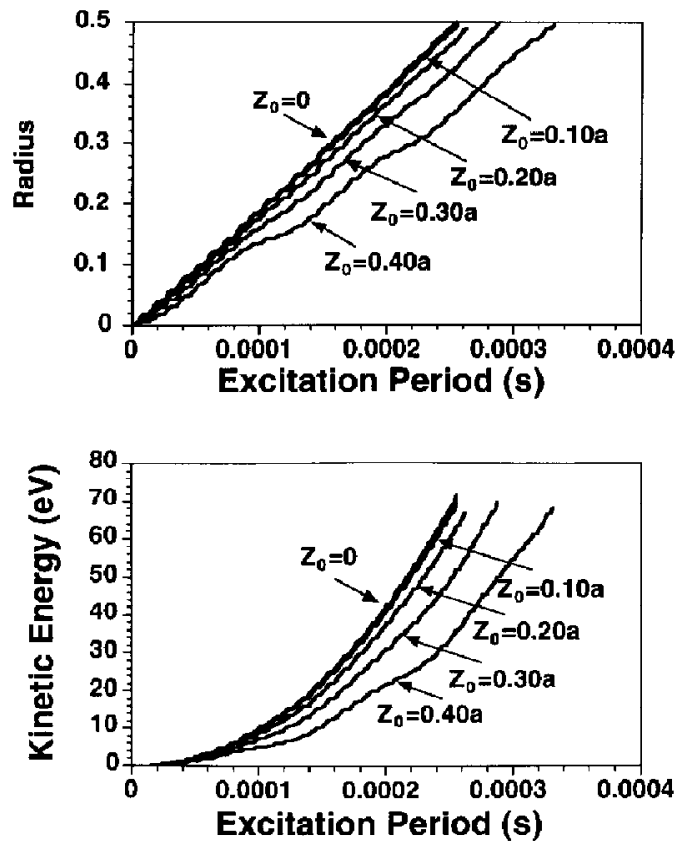

Figure 3. Temporal variation of (a) instantaneous radius and (b) kinetic energy of an ion of $m / z 1000$ initially on the $z$ axis, for each of several initial $z$ displacements during single-frequency on-resonance excitation electric field $10 / a \mathrm{~V}(\mathrm{p}-\mathrm{p}) / \mathrm{m}$. The ion radius is $\sqrt{x^{2}+y^{2}}$ and kinetic energy is $m \dot{r}^{2} / 2$.

one cycle of the magnetron motion is longer than the maximum displayed excitation period.) On average, ion radius increases approximately linearly with excitation period for ions initially at rest at the center of the trap and increases more slowly for ions of finite initial $z$ displacement. Thus because $z$ oscillation frequency varies as $1 / \sqrt{m}$, dipolar excitation at $\omega_{+}$could result in mass-to-charge ratio dependent postexcitation ion radii if the excitation amplitude is high enough such that the excitation event is shorter than one $z$ oscillation period.

Yet another view of incoherent excitation is provided by Figure 4, which shows that the postexcitation ion radius decreases with increasing initial ion $z$ displacement for an ion of $\mathrm{m} / z 1000$ during singlefrequency on-resonance excitation. As the excitation period increases, the spread in average radius among ions of different initial $z$ displacement increases.

\section{Effects of z Displacement on Detected ICR Signal}

Figure 5 shows how the detected ICR signal varies with initial $z$ displacement of an ion of $m / z 1000$. The ICR signal detected from such excitation is reduced in two ways compared to that for ions excited and detected with uniform rf electric field: (1) ions of larger initial $z$ displacement are excited to lower cyclotron radius and thus induce a smaller-amplitude signal and

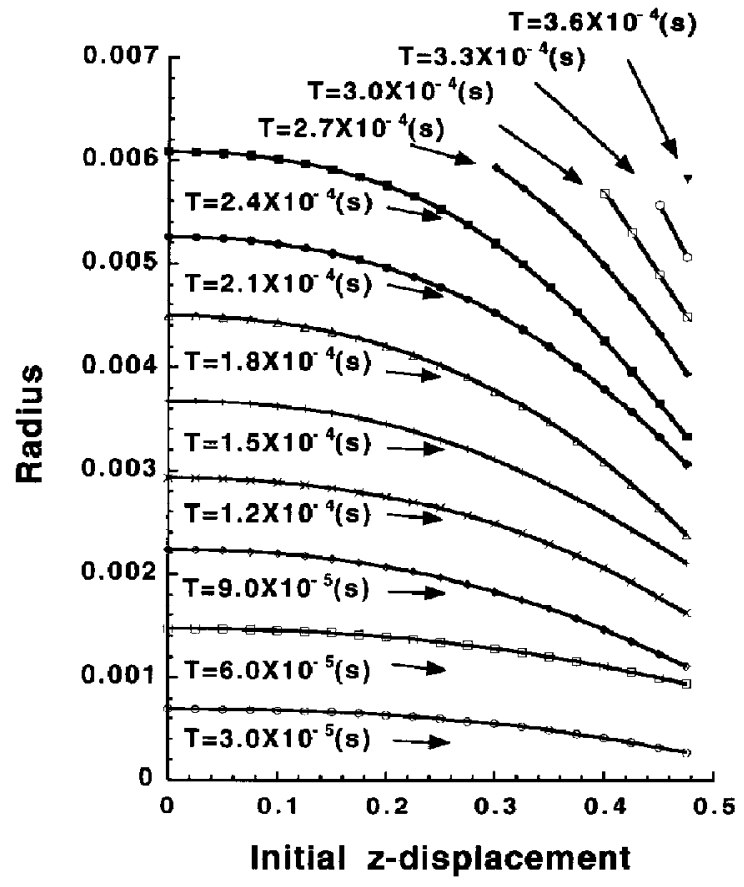

Figure 4. Average ion radius versus initial $z$ displacement for an ion of $m / z 1000$ initially on the $z$ axis, following singlefrequency on-resonance excitation electric field $10 / \pi \mathrm{V}\left(\mathrm{p}^{-} \mathrm{p}\right) / \mathrm{m}$. $T$ is the excitation period. The average ion radius is the instantaneous radius $\left(\sqrt{x^{2}+y^{2}}\right)$ averaged over many magnetron cycles.

(2) ions of different initial $z$ displacement end up with different cyclotron orbital phase, which results in some destructive interference that further reduces the detectable ICR signal. Thus the detected ICR signal (Figure 5) increases less rapidly than the average radius of the ions themselves (Figure 4); for example, after an excitation period of $2.7 \times 10^{-4} \mathrm{~s}$, the signal for ions initially at $z=0.45 a$ is approximately one third that of ions initially at $z=0$, whereas the average radius for ions of $z_{0}=0.45 a$ is approximately $60 \%$ of that for ions initially at $z=0$.

Figure 6a shows two simple ion $z$ distributions (spatially uniform and Gaussian) from which we can begin to characterize the effect of $z$ distribution on ICR behavior in a cubic ICR ion trap. ICR spectra calculated from each ion distribution are shown in Figure $6 b$. The spectrum for initially Gaussian-distributed ions has less pronounced side bands at $\omega_{+} \pm 2 \omega_{z}$ than are seen for an initially uniform ion $z$ distribution, because most of the Gaussian-distributed ions remain close to the trap midplane during excitation $\mathrm{A}$ signal at the third-harmonic frequency $3 \omega_{+}$is seen in both spectra.

The signal magnitude at frequency $\omega_{+}$from spectra of the type shown in Figure 6 is displayed as a function of excitation period in Figure 7. The ICR signal for 


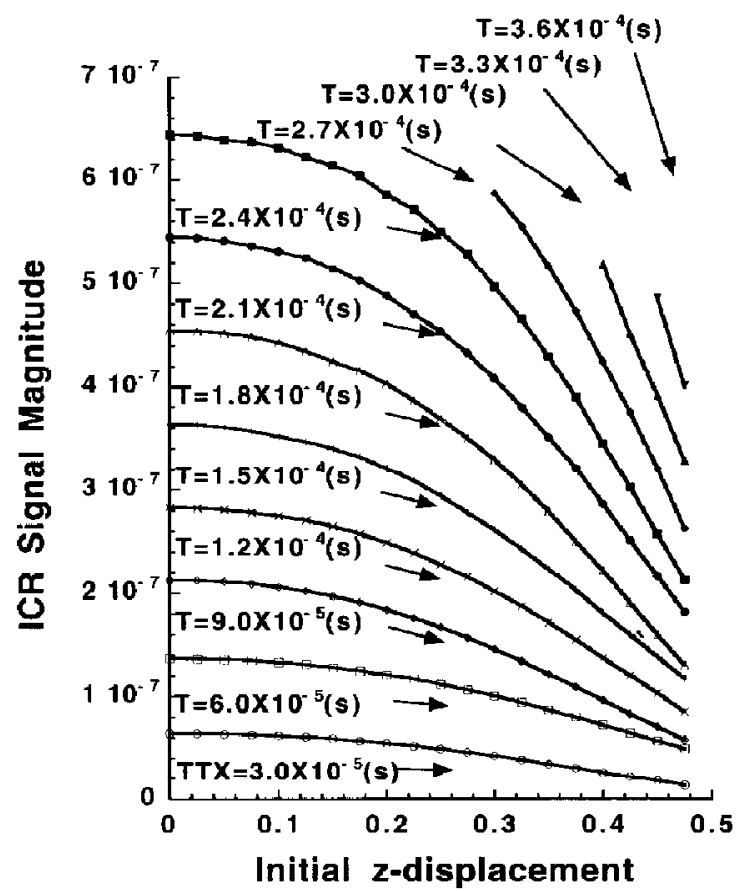

Figure 5. ICR signal magnitude at frequency $\omega_{+}$versus initial ion $z$ displacement for an ion of $m / z 1000$ initially on the $z$ axis, following single-frequency on-resonance excitation electric field $10 / a \mathrm{~V}(\mathrm{p}-\mathrm{p}) / \mathrm{m} . T$ is the excitation period. initially Gaussian-distributed ions rises faster and to a higher magnitude during resonant excitation than does the signal for initially uniformly distributed ions, and also drops to zero more rapidly after the onset of radial ejection, because most of the Gaussiandistributed ions remain near the $z=0$ trap midplane where the excitation field is strongest. The highest signal and most nearly simultaneous ejection would result if all ions were located at $z=0$ initially.

Ejection of Ions at $2 \omega_{z}$ and $\omega_{+}+2 \omega_{z}$

Experimentally it is well known that ions are ejected at $\omega_{+}+2 \omega_{z}$ during broadband excitation because of coupling between radial and $z$ components of the excitation electric field [12-19]. $z$ ejection is highly undesirable because relative FT-ICR mass spectral peak magnitudes of ions of different mass-to-charge ratio depend on the excitation amplitude-duration product, thereby distorting apparent ion relative abundances in an FT-ICR mass spectrum. Although $z$ ejection may be effectively eliminated by appropriate "shimming" of the excitation electric field $[17,23,24], z$ ejection remains a potentially severe problem for more conventional cubic, tetragonal, and cylindrical ion traps.

Both previous theoretical and experimental results illustrate that ion loss due to $z$ ejection is more pronounced for low-to-high than for high-to-low frequency-sweep excitation, because in a low-to-high frequency sweep, ions are first excited off-axis where the excitation field $z$ component is larger, followed by

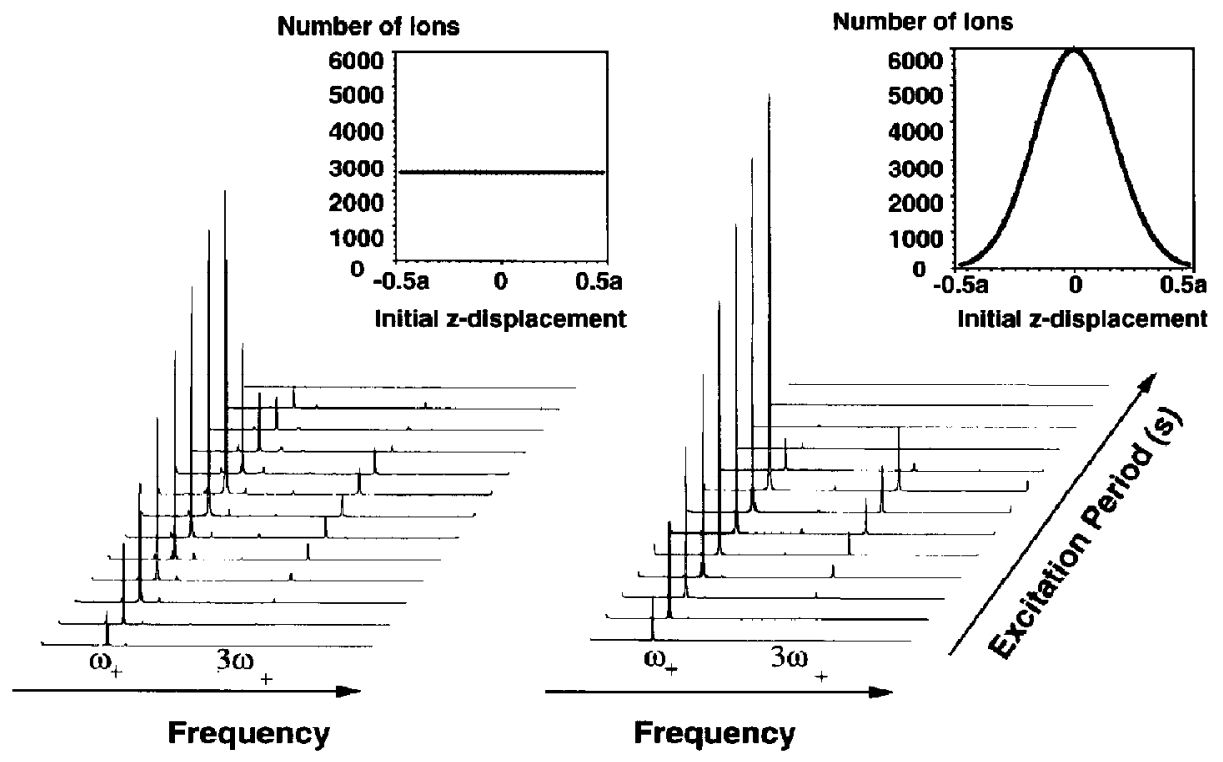

Figure 6. Simulated FT-ICR frequency-domain spectra for 99,648 ions of $m / z 1000$ distributed either (a) uniformly or (b) Gaussian distributed along the $z$ axis following various excitation periods from 3.0 to $3.9 \times 10^{-4} \mathrm{~s}$ at increments of $3.0 \times 10^{-5} \mathrm{~s}$. The standard deviation $\sigma$ for the Gaussian distribution is $a / 6$, with a mean value of 0 . The value 99,648 represents $99.7 \%$ of $10^{5}$ ions located within $\pm 3 \sigma- \pm a / 2$ of the trap midplane. 


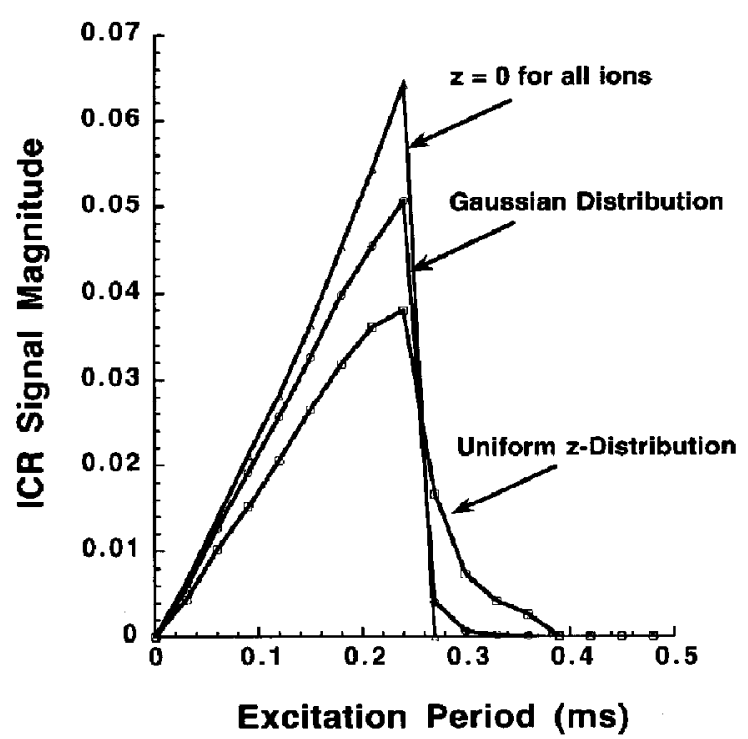

Figure 7. Calculated ICR signal magnitude at frequency $\omega_{+}$ versus excitation period of 99,648 ions of $m / z$ 1000. Top: ions all initially located at $z=0$; middle: Gaussian initial $z$ distribution; bottom: ions initially uniformly distributed along the $z$ axis.

excitation of the $z$ oscillation at $\left(\omega_{+}+2 \omega_{z}\right)$. However, in typical FT-ICR experiments, most ions are formed or injected on or near the $z$ axis where $E_{z}$ is zero by symmetry. As a result, ions should experience little or no $z$ excitation at $2 \omega_{z}$. However, we and others have observed experimentally that significant ion loss occurs by excitation at $2 \omega_{z}$ and $\omega_{+}+2 \omega_{z}$. The present numerical simulations offer the first direct theoretical demonstration of those effects. Figure 8 shows ion trajectories during single-frequency excitation at $2 \omega_{z}$
Table 1. Excitation period leading to onset of radial ejection following on-resonance single-frequency dipolar excitation ${ }^{\star}$

\begin{tabular}{lccc}
\hline & \multicolumn{3}{c}{ Ejection onset } \\
\cline { 2 - 4 } $\mathrm{m} / \mathrm{z}$ & $\begin{array}{c}\text { Excitation at } \\
\omega_{+}\left(\times 10^{-4} \mathrm{~s}\right)\end{array}$ & $\begin{array}{c}\text { Excitation at } \\
2 \omega_{z}(\mathrm{~s})\end{array}$ & $\begin{array}{c}\text { Excitation at } \\
\omega_{+}+2 \omega_{z}\left(\times 10^{-4} \mathrm{~s}\right)\end{array}$ \\
\hline \hline 1000 & 2.8 & $7.1 \times 10^{-4}$ & 6.4 \\
2000 & 2.7 & $8.5 \times 10^{-4}$ & 7.6 \\
3000 & 2.8 & $1.5 \times 10^{-3}$ & 9.2 \\
4000 & 2.6 & $1.5 \times 10^{-3}$ & 8.9 \\
5000 & 2.7 & $1.1 \times 10^{-3}$ & 9.8 \\
\hline
\end{tabular}

Excitation electric field amplitude $10 / a \mathrm{~V}(\mathrm{p}-\mathrm{p}) / \mathrm{m}$ at excitation frequencies $\omega_{+}, 2 \omega_{z}$, and $\omega_{+}+2 \omega_{z}$; ion $\mathrm{m} / z$ 5000; ion initial position $(0,0,0.25 a)$, in which $a$ is the length of one side of the cubic ICR ion trap, and ion initial velocity is zero.

(Figure 8a) and at $\omega_{+}+2 \omega_{z}$ (Figure 8b) for initially on-axis ions of $m / z 1000$. We see that ions are first pushed away from the $z$ axis due to off-resonant excitation [11]; then once the ions are off-axis, dipolar excitation at $2 \omega_{z}$ excites $z$ oscillation at $\omega_{z}[1]$ and the ions eventually may be ejected axially. Table 1 lists the excitation period leading to onset of ejection during single-frequency excitation at frequencies $\omega_{+}$(radial ejection), $2 \omega_{z}$ (axial ejection), and $\omega_{+}+2 \omega_{z}$ (radial ejection) for ions of $1000 \leq m / z \leq 5000$ with initial position $(0,0$, and $0.25 a)$. The time required to reach the onset of radial ejection by dipolar excitation at $\omega_{+}$ is about four to five times shorter than it takes to eject ions axially by dipolar excitation at $\omega_{+}+2 \omega_{z}$ or $2 \omega_{z}$.

From the foregoing analysis, it becomes clear that all of the (generally undesirable) nonlinear effects are minimized if initial ion $z$ displacement is small. Because the $z$ component of the electrostatic ("trapping") potential is approximately quadratic, ions that undergo repeated collisions with neutrals will ultimately lose kinetic energy and relax toward the $z=0$ mid-
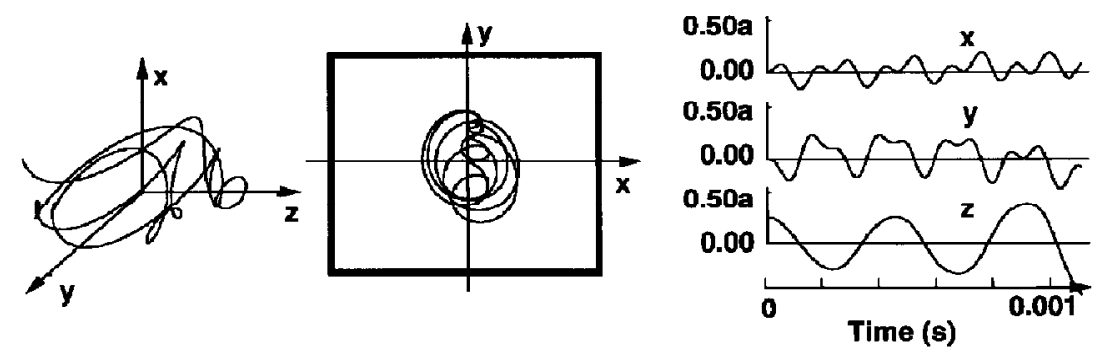

Figure 8. Various ion trajectory projections during radial dipolar single-frequency on-resonance excitation of amplitude $10 / a \quad \mathrm{~V}$ $(\mathrm{p}-\mathrm{p}) / \mathrm{m}$, at frequency (a) $2 \mathrm{\omega}_{z}$ and (b) $\omega_{+}+2 \omega_{z}$ for ions of $m / z 5000$; initial position $(0,0$, and $0.25 a)$. Left: three-dimensional display; middle: projection of the ion trajectory onto the $z=0$ midplane; right: temporal variation of ion $x$, $y$, and $z$ coordinates.
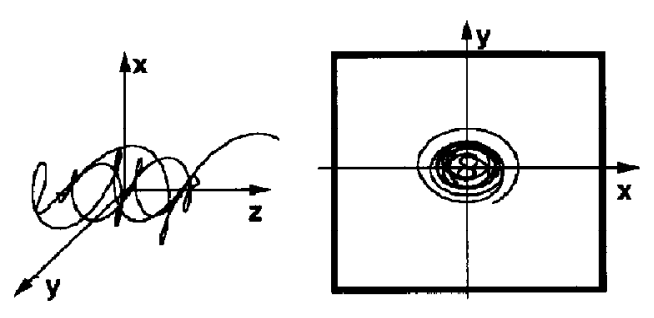
plane of the trap. However, the ion magnetron radius must increase (at a slower rate) for the same reason [25], so that the ions will eventually be lost by collisions with the side plates. Nevertheless, ions may be brought to the trap center by ion-neutral collisions without an increase in magnetron radius by converting magnetron to cyclotron motion by use of azimuthal quadrupolar excitation at the unperturbed cyclotron frequency $\omega_{c}=q B / m$ (S.I. units), in which $m$ and $q$ are ion mass and charge, and $B$ is applied magnetic field induction [26-28], or by converting magnetron (and cyclotron) motion to axial motion by quadrupolar excitation of $x z$ symmetry at the appropriate frequency [25]. Because the cyclotron radius or $z$-oscillation amplitude decreases faster than the magnetron radius increases as a result of ion-neutral collisions, the net effect is that ions all eventually relax to the center of the trap.

\section{Acknowledgments}

This work was supported by the National Science Foundation (CHE-90-21058), the Public Health Service (NIH GM-31683), The Ohio State University, and the National High Magnetic Field Laboratory at Florida State University. The authors deeply appreciate Dr. S. Guan's helpful discussions and help for stack-plotting spectra for the uniform and Gaussian distributions. The Ohio Supercomputer Center and Ohio Visualization Lab provided the computing facilities.

\section{References}

1. Schweikhard, L.; Marshall, A. G. I. Am. Soc. Mass Spectrom. 1993, 4, 433-452.

2. Comisarow, M. B. I. Chem. Phys. 1978, 69, 4097-4104.

3. Comisarow, M. B. In Ion Cyclotron Resonance Spectrometry II; Hartmann, H,; Wanczek, K.-P., Ed.; Springer: Berlin, 1982; PP 484-513.

4. Limbach, P. A.; Grosshans, P. B.; Marshall, A. G. Anal. Chem. $1993,65,135-140$.

5. Williams, E. R.; Henry, K. D.; McLafferty, F. W. J. Am. Chem. Soc. 1990, 172, 6157-6162.
6. Speir, J. P.; Gorman, G. S.; Pitsenberger, C. C.; Turner, C. A.; Wang, P. P.; Amster, I. J. Anal. Chem. 1993, 65, 1746-1752.

7. Guan, Z.; Hoftstadler, S. A.; Laude, D. A., Jr. Anal. Chem. 1993, 65, 1588-1593.

8. Guan, S. J. Am. Soc. Mass Spectrom. 1991, 2, 483-486.

9. Grosshans, P. B.; Chen, R.; Marshall, A. G. Int. I. Mass Spectrom. Ion Processes 1994, in press.

10. Brown, L. S.; Gabrielse, G. Rev. Mod. Phys. 1986, 58, 233-311.

11. Wang, M.; Marshall, A. G. Int. J. Mass Spectrom. Ion Processes $1990,100,323-346$.

12. Huang, S. K.; Rempel, D. L.; Gross, M. L. Int. J. Mass Spectrom. Ion Processes 1986, 72, $15-31$.

13. Rempel, D. L.; Huang, S. K.; Gross, M. L. Int. J. Mass Spectrom. Ion Processes 1986, 70, 163-184.

14. Allemann, M.; Kofel, P.; Kellerhals, H.; Wanczek, K-P. Int. J. Mass Spectrom. Ior Processes 1987, 75, 47-54.

15. van der Hart, W. J.; van de Guchte, W. J. Int. I. Mass Spectrom. Ion Processes 1988, 82, 17-31.

16. van de Guchte, W. J.; van der Hart, W. J. Int. J. Mass Spectrom. Ion Pracesses 1990, 95, 317-326.

17. Wang, M.; Marshall, A. G. Anal. Chem. 1990, 62, 515-520.

18. Fujiwara, M.; Yamauchi, T.; Miura, K.; Inoue, M. J. Amer. Soc. Mass Spectrom. 1992, 3, 847-852.

19. Mitchell, D. W.; Hearn, B. A.; DeLong, S. E. Int. I. Mass Spectrom. Ion Processes 1993, 125, 95-126.

20. Dunbar, R. C. Int. J. Mass Spectrom. Ion Processes 1984, 56, 1.

21. Grosshans, P. B.; Shields, P. J.; Marshall, A. G. I. Chem. Phys. 1991, 94, 5341-5352.

22. Xiang, X.; Guan, S.; Marshall, A. G. I. Am. Sac. Mass Spectrom. 1994, 8, 238-249.

23. Hanson, C. D.; Castro, M. E.; Kerley, E. L.; Russell, D. H. Anal. Chem. 1990, 62, 520-526.

24. Caravatti, P.; Allemann, M. Org. Mass Spectrom. 1991, 26. 514-518.

25. Guan, S; Xiang, X.; Marshall, A. G. Int. J. Mass Spectrom. Ion Processes 1993, 124, 53-67.

26. Schweikhard, L.; Guan, S.; Marshall, A. G. Int. I. Mass Spectrom. Ion Processes 1992, 120, 71-83.

27. Bollen, G.; Moore, R. B.; Savard, G.; Stolzenberg, H. Appl. Phys. 1990, 68, 4355-4374.

28. Savard, G.; Becker, S.; Bollen, G.; Kluge, H.-J.; Moore, R. B.; Schweikhard, L.; Stolzenberg, H.; Wiess, U. Phys. Lett. A 1991, 158, 247-252. 